\title{
EchoGéo
}

21 | 2012

Pays émergents

\section{" Đi chơi đi ! ". « Allons-nous amuser !»}

Entre public et privé, une approche socio-spatiale des pratiques de loisirs à Hồ Chí Minh Ville

\section{Emmanuelle Peyvel et Marie Gibert}

\section{(2) OpenEdition}

\section{Journals}

\section{Édition électronique}

URL : https://journals.openedition.org/echogeo/13177

DOI : $10.4000 /$ echogeo.13177

ISSN : 1963-1197

\section{Éditeur}

Pôle de recherche pour l'organisation et la diffusion de l'information géographique (CNRS UMR 8586)

\section{Référence électronique}

Emmanuelle Peyvel et Marie Gibert, «" Đi chơi đi ! ". « Allons-nous amuser ! » », EchoGéo [En ligne], 21 | 2012, mis en ligne le 10 octobre 2012, consulté le 10 août 2021. URL : http://journals.openedition.org/ echogeo/13177 ; DOI : https://doi.org/10.4000/echogeo.13177

Ce document a été généré automatiquement le 10 août 2021.

EchoGéo est mis à disposition selon les termes de la licence Creative Commons Attribution - Pas d'Utilisation Commerciale - Pas de Modification 4.0 International (CC BY-NC-ND) 


\section{" Đi chơi đi ! ". « Allons-nous amuser!»}

Entre public et privé, une approche socio-spatiale des pratiques de loisirs à Hồ Chí Minh Ville

\section{Emmanuelle Peyvel et Marie Gibert}

1 A l'ombre des BRIC, le Việt Nam s'affirme de plus en plus comme pays émergent avec sa moyenne de $7 \%$ de croissance annuelle depuis 2001 , soit la deuxième plus importante en Asie après la Chine. Ce jeune marché de 85,8 millions de consommateurs - d'un âge médian de 28 ans, selon le dernier recensement de la population - massivement éduqués et soignés ${ }^{1}$, attire de plus en plus les IDE, qui représentent aujourd'hui 35,8\% du PIB (État du monde, 2010). Cette bonne santé économique va de pair avec une visibilité accrue sur la scène internationale, marquée par l'adhésion du pays en 1995 à l'ASEAN, en 1998 à l'APEC et en 2007 à l'OMC. Ces chiffres et ces dates alimentent une approche du pays en termes d'émergence et de transition dans nombre de travaux scientifiques ${ }^{2}$. Cette vision rejoint la rhétorique du « Renouveau » (Đổi Mới) développée par l'État depuis le $6^{e}$ Congrès du Parti Communiste vietnamien en 1986, afin de légitimer le passage à une " économie de marché à orientation socialiste ». Cependant, cette convergence de points de vue, a priori paradoxale, ne doit pas se transformer en une grille de lecture simplificatrice, renvoyant dos à dos un avant et un après.

2 Forçant à s'inscrire dans une temporalité plus complexe, l'analyse socio-spatiale des pratiques de loisirs, ici menée à travers le cas de Hồ Chí Minh Ville, nous permet d'envisager la notion d'émergence de manière originale. Ce thème est encore peu étudié dans les rares travaux portant sur les productions et pratiques culturelles dans les villes vietnamiennes (Drummond et Thomas, 2003 ; Earl, 2004 ; Harms, 2009). Les loisirs constituent pourtant une façon concrète d'approcher les usages de la richesse nouvellement produite, sa répartition et le sens qui lui est affecté.

3 Par « loisirs ", nous entendons toute activité à laquelle l'individu s'adonne par choix, afin d'atteindre au moins un de ces buts : le délassement (temps de récupération), le divertissement (s'opposant au temps de travail répétitif) et le développement (participation active à la culture) (Dumazedier, 1962). Contrairement au tourisme, ces 
activités ont lieu dans l'espace-temps du quotidien (Knafou, 1997). Le présent article ne cherche pas à définir ce que sont les loisirs à l'aune du cas vietnamien, mais plutôt de comprendre ce que les Vietnamiens entendent par loisirs. Plus qu'une catégorie strictement scientifique, les loisirs sont ici envisagés selon la définition vernaculaire qu'en font nos enquêtés, sans reprendre des débats plus théoriques sur le repos, considéré tantôt comme appartenant à la sphère du travail (Marx, 1894), tantôt comme un temps libre (Dumazedier, 1962; Lanfant, 1972); ou sur les pratiques de consommation, que certains auteurs ne considèrent pas comme faisant partie d'un temps véritablement libre (Baudrillard, 1970). Dans une perspective de géographie sociale de l'émergence, nous étudierons les dimensions spatiales des pratiques de loisirs, en nous efforçant de dépasser une approche économique, qui envisage les loisirs comme un marché.

4 La pratique des loisirs est d'abord et surtout une affaire de citadins (Dumazedier, 1966; Pinol, 1992; Bourillon, 2000 ; Delattre, 2000). Ces derniers constituent un vivier rentable, du fait de leur concentration et de leurs moyens, dans un lieu d'ouverture, favorable aux innovations. Locomotive économique du pays, Hồ Chí Minh Ville constitue en cela un terrain d'étude privilégié au Việt Nam. Avec ses huit millions d'habitants officiellement recensés ${ }^{3}$, cette métropole émergente a historiquement bâti sa puissance sur les activités commerciales, propices aux brassages de populations. D'abord comptoir chinois de Chợ Lớn, elle devient perle de l'Orient colonial puis base arrière américaine pendant une vingtaine d'années. Aujourd'hui, Hồ Chí Minh Ville est la ville vietnamienne la plus insérée dans la mondialisation, processus favorisant la circulation et l'hybridation de nouveaux modèles sociaux et culturels. Nous posons comme hypothèse que les pratiques de loisirs aboutissent à une différenciation accrue des espaces publics et privés depuis le Đổi Mới. Entraînant à la fois une libéralisation encadrée de l'économie et un assouplissement du contrôle social, cette politique tolère de nouveau propriété privée et société de consommation, processus inhérents à l'émergence. En cela, les loisirs constituent une clé de lecture stimulante pour appréhender les recompositions socio-spatiales à l'œuvre dans le processus d'émergence. En outre, ils apparaissent comme un bon moyen d'analyser la négociation s'opérant entre une société confucéenne et socialiste, où la collectivité et la famille sont structurantes, et des citadins aspirant aujourd'hui à plus de libertés individuelles. De cette tension résulte une recomposition des rapports entre public et privé, collectif et individuel, promiscuité et intimité, contrainte et plaisir. Nous proposons de lire ces dynamiques à l'œuvre dans leurs dimensions spatiales, à partir des pratiques et des lieux de loisirs à Hồ Chí Minh Ville.

5 Pour répondre à ces interrogations, nous avons privilégié une démarche qualitative fondée sur l'observation participante et la conduite d'entretiens semi-directifs, à la faveur d'une présence prolongée sur le terrain de plusieurs années. Notre ambition était d'approcher les pratiques in situ, leur déploiement spatial et le sens qui leur est donné. Pour ce faire, une quinzaine d'entretiens approfondis ont été conduits auprès de Vietnamiens que nous avons suivis dans leurs pratiques de loisir (cours de yoga, sorties entre amis, karaoké, shopping...). Nous nous sommes concentrées sur une population jeune (entre 20 et 35 ans), active ou en passe de le devenir, et surtout première génération à bénéficier de la stabilité et de la prospérité induites par le Đổi Mới. Ces entretiens ont été complétés par des observations participantes menées dans des lieux variés de loisirs (cafés, parc d'attractions, club de golf...). Combinées, ces deux outils nous ont permis de replacer les loisirs dans une certaine cohérence temporelle, au-delà 
des pratiques ponctuelles, afin de mesurer leur importance, leur fréquence et leur articulation avec le temps de travail. L'objectif n'était pas de recenser exhaustivement tous les lieux de loisirs de la ville, mais bien de comprendre la place qui leur est accordée à travers leurs dynamiques spatiales.

6 Nous envisagerons tout d'abord l'ouverture comme condition première d'apparition d'une sphère privée du bon temps, avant d'analyser la question de l'accès aux loisirs, en étroite relation avec les mobilités intra-urbaines. Enfin, la question de la privatisation des loisirs nous conduira à réinterroger les processus d'ouverture et de fermeture spatiales à l'œuvre à Hồ Chí Minh Ville.

\section{L'ouverture comme condition d'émergence des loisirs à Hồ Chí Minh Ville}

\section{Approcher l'émergence par les emplois du temps}

7 Hồ Chí Minh Ville est aujourd'hui la capitale économique incontestée du pays. En 2011, son taux de croissance économique était de $10,2 \%$, soit le double de celui national (CBRE, 2011). Ce dynamisme économique se combine avec une forte croissance démographique, estimée à $34,7 \%$ entre 2001 et 2010. Sa population travaille essentiellement dans le secteur tertiaire et secondaire, représentant respectivement $53,6 \%$ et $45,3 \%$ de la structure économique de la ville en 2010 (Ho Chi Minh City Statistical office, 2010). Ce fonctionnement économique s'est accompagné d'une montée en puissance du salariat. Aujourd'hui, c'est près d'un actif sur deux qui y est salarié (DIAL, 2008). Ainsi régie par le code du travail, son activité est limitée à 8 heures par jour et 48 heures par semaine (chapitre VII, article 68), cette durée pouvant être abaissée pour des métiers très pénibles. En outre, d'après l'article 72, «le salarié $a$ droit à un repos hebdomadaire d'une durée minimale de 24 heures consécutives » ou de quatre jours par mois, si ce repos hebdomadaire est impossible, auquel s'ajoute en moyenne une douzaine de jours de congés annuels.

8 Si ces chiffres sont cependant à nuancer par l'importance de l'économie informelle et les entorses au code du travail, la tendance est bien là : les habitants de Hồ Chí Minh Ville sont de plus en plus nombreux et riches, grâce à une économie tertiarisée et industrialisée, salariant de manière croissante sa main-d'œuvre. Ces éléments sont justement ceux constituant les conditions les plus favorables à l'émergence d'une sphère récréative. En effet, le salariat introduit des changements durables dans la gestion du temps. Le contrat hebdomadaire voire mensuel garantit une régularité non seulement de revenus mais aussi d'emploi du temps, séparant plus strictement temps de travail et temps libre, ce qui est propice à l'organisation de projets récréatifs. Si on travaille encore tous les jours à Hồ Chí Minh Ville, un week-end est en train de se structurer, davantage dévolu au repos et aux distractions (Gubry et alii, 2008). Cet état de fait est important à souligner, car il permet de complexifier l'approche de la classe moyenne, habituellement définie par ses revenus, alors que les seuils retenus ne font pas consensus et que la notion même est discutée (Gainsborough, 2002 ; King, 2008). S'il est vrai que les Vietnamiens ont de plus en plus d'argent, ils ont aussi et surtout plus de temps pour en profiter. 


\section{Les loisirs, entre investissements étatiques et privés}

9 Le marché des loisirs est aujourd'hui investi par des acteurs économiques aux statuts variés, ce qui n'était pas envisageable avant 1986. L'État exerçait alors un monopole sur l'organisation des loisirs et du tourisme. Conçus comme un outil d'émulation sociale, les loisirs constituaient une récompense à destination des ouvriers, paysans et fonctionnaires désignés comme les plus méritants. Ne correspondant pas à un secteur économique en tant que tel, les loisirs ne répondaient alors à aucune logique de rentabilité. Cet interventionnisme est renégocié avec l'ouverture. Cette dernière s'est traduite par un désengagement graduel de l'État, qui en a cependant orchestré les modalités de mise en œuvre.

Les entreprises et investissements étatiques sont progressivement recentrés sur les activités jugées les plus stratégiques et rentables, dont certaines sont directement liées au développement d'une sphère récréative. La holding Saigon Tourism, créée en 1975 et refondée en 1999 sous l'égide du comité populaire de la ville, incarne bien cette recomposition des modes d'action étatique. Cette société investit tour à tour en tant que propriétaire, gestionnaire ou investisseur dans la restauration, l'hébergement, les transports, le sport et la culture. Dotée d'un capital équivalent à plus de 126 millions d'euros, elle est aujourd'hui leader sur le marché national. Elle coopère aujourd'hui de plus en plus avec des entreprises privées, vietnamiennes comme étrangères, dans la conduite de ses activités.

11 Cette recomposition du secteur public va de pair avec la montée en puissance d'acteurs privés. Tel est le cas de Đạt Hoàng, directeur du club Yoga living, localisé en plein centre ville. Cet entrepreneur, âgé d'une trentaine d'années, tire partie de l'ouverture du pays : après des études à l'université de Wasada (Japon), à la suite desquelles il a opté pour le pseudonyme de "Dato ", il s'est inscrit en management à la RMIT, université australienne implantée à Hồ Chí Minh Ville depuis 2001. Se définissant comme un " aventurier capitaliste ", il s'est lancé simultanément dans l'immobilier et la création d'un club de yoga. Pour attirer des clients, il joue de deux stratégies complémentaires. D'une part, via son site internet et ses réseaux personnels, il s'adresse à une population aisée, locale comme expatriée, issue du monde des affaires, en proposant des services aux prix élevés. D'autre part, il propose des abonnements à destination des entreprises vietnamiennes qui les offrent à leurs employés, via leurs syndicats hérités de l'époque socialiste. Cet exemple montre la diversité des stratégies de conquête du marché des loisirs par les acteurs privés, jouant à la fois de logiques individuelles et collectives. La fréquentation simultanée de populations socialement différenciée amène cependant Dato à des arrangements spatiaux et temporels, avec des salles de cours séparées et des plannings différents. Le développement contemporain du secteur des loisirs à Hồ Chí Minh Ville conduit ainsi à une stratification complexe des acteurs publics et privés.

\section{L'ouverture à des modèles mondialisés de loisirs}

Les loisirs sont choses anciennes au Việt Nam. Fruits des circulations avec le monde chinois, des pratiques récréatives s'épanouissent à la cour impériale, se combinant à d'autres plus populaires : jeux - comme le mah-jong, la balançoire ou le cerf-volant danses, chants, musique, poésie et contes, ou encore paris sur animaux (Schweyer, 2005, Durand, 2011). Toutefois, il serait anachronique de parler à cette époque d'une 
structuration formalisée de la sphère des loisirs. En revanche, il est intéressant de noter non seulement l'ancienneté de ces pratiques, mais aussi la façon dont elles ont pu s'hybrider avec d'autres inédites, apparues avec la colonisation française (exemple de l'hippodrome réactivant les paris sur animaux). L'élite locale les intègre en partie, comme le tennis ou la baignade; ce qui a pu être tantôt moqué (Vũ, 1936) tantôt valorisé comme signe de « Progrès » (Nguyễn, 1995). L'arrimage au monde socialiste du pays constitue une seconde mondialisation, sur le modèle soviétique. Mobilisée au service de "l'édification de l'Homme nouveau", la sphère des loisirs participe en théorie d'une justice sociale, à l'image du "cercle européen " (cf. illustration 1). Les ouvriers et employés désormais accueillis peuvent y voir des expositions et s'essayer aux sports, à la lecture, à la musique et au théâtre.

Illustration 1 - La requalification socialiste du cercle de loisirs européen à Hồ Chí Minh Ville

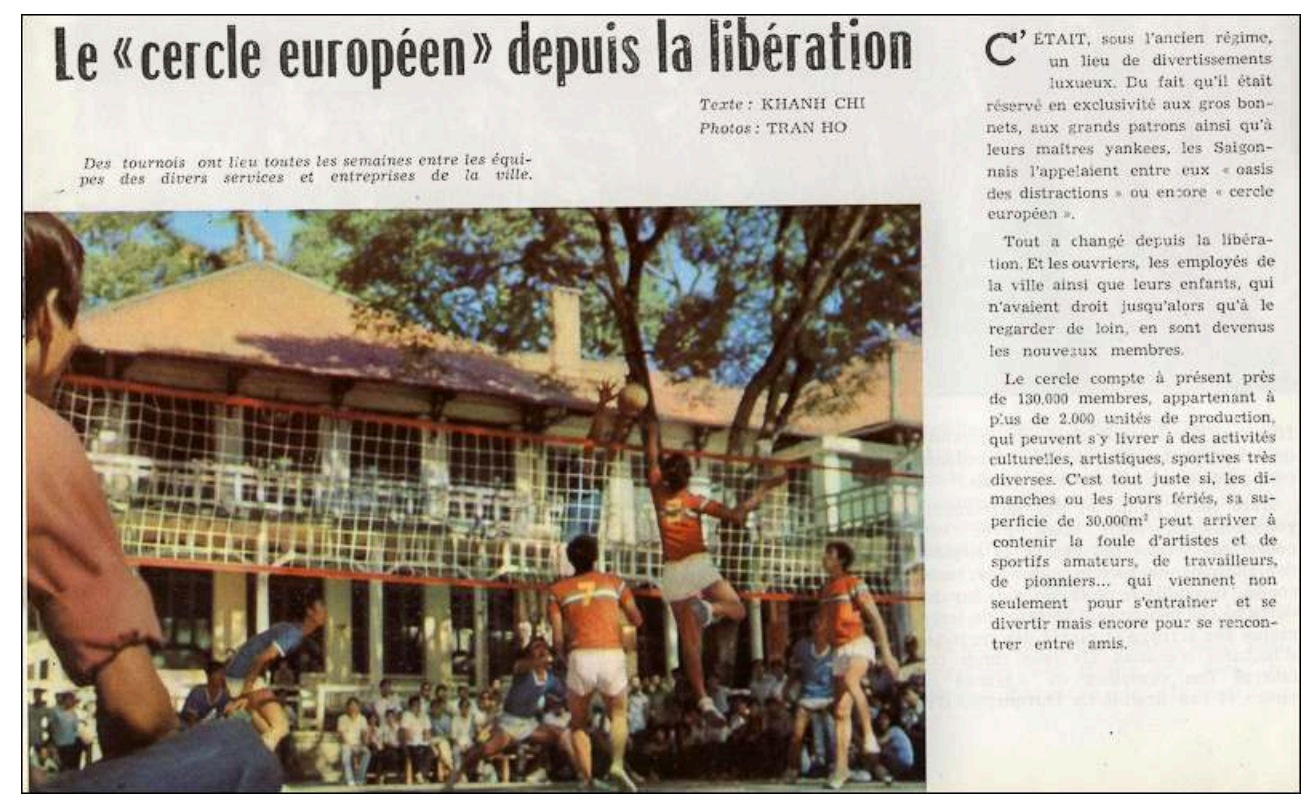

Source : Khanh et Tran, 1978.

A partir du Đổi Mới, la sphère des loisirs est largement investie par des pratiques de consommation (Drummond et Thomas, 2004). La construction de centres commerciaux et de malls ${ }^{4}$ aux standards internationaux est un important marqueur urbain des années 2000 dans le centre de Hồ Chí Minh Ville. C'est en 1997 que fut ouvert le premier, Saigon Center, dans le district 1, suivi en 1999 du Diamond Plaza, stratégiquement localisé derrière la cathédrale. Différents malls de la chaîne malaisienne Parkson furent également implantés dans le centre-ville entre 2005 et 2009. L'ouverture en $2010 \mathrm{du}$ Vincom Center marque un tournant par l'échelle du bâtiment érigé dans l'emblématique rue Đồng Khởi. On compte ainsi une dizaine de grands malls à Hồ Chí Minh Ville aujourd'hui, tous situés dans l'hypercentre ou dans les nouvelles centralités périphériques comme Saigon South (Saigon Paragon construit en 2009 et Crescent Mall en cours de construction). La fréquentation de ces malls est vécue comme une expérience urbaine de la "modernité ", systématiquement assimilée à un loisir par nos enquêtés. Les centres commerciaux deviennent des lieux de sociabilité et de promenade. La période de Noël est particulièrement révélatrice de ce phénomène, avec la 
déambulation de centaines de familles et groupes d'amis le long des vitrines des enseignes étrangères du centre ville (illustration 2).

Illustration 2 - Loisirs et émergence d'une société de consommation mondialisée à Hồ Chí Minh Ville

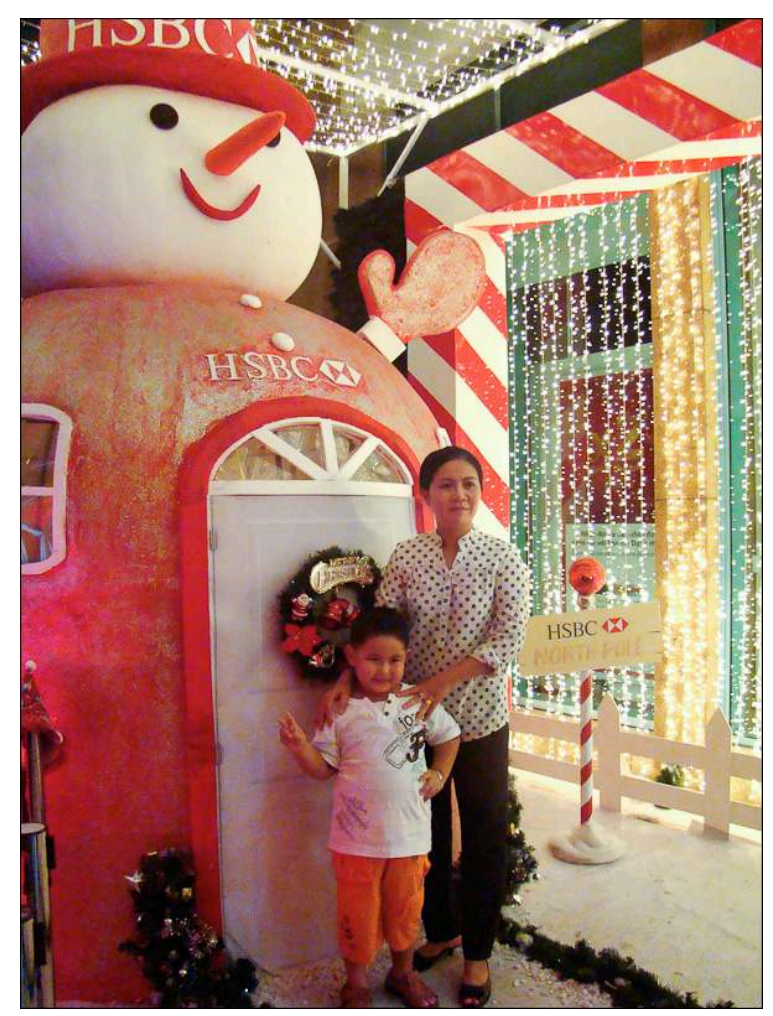

Auteur : M. Gibert, décembre 2010. banque chinoise mondialisée, HSBC, dont les décors sont largement investis par les badauds venus s'y promener et s'y photographier dans une ambiance festive. Cependant, ces malls en apparence ouverts, restent discriminants socialement. En grande majorité situés dans le centre-ville, ils sont à la fois facteurs et marqueurs de centralité métropolitaine. En ce sens, ils participent de dynamiques socio-spatiales similaires à d'autres équipements de prestige, comme les grands hôtels (Sanjuan, 2003).

\section{Se déplacer, se récréer : l'accès aux loisirs à Hồ Chí Minh Ville}

\section{Multiplication des lieux et des mobilités de loisirs dans la ville}

Les mobilités intra-urbaines ont récemment explosé à Hồ Chí Minh Ville (Truitt, 2008). De 1990 à 2000, le marché des deux roues motorisées a connu une croissance moyenne annuelle de $6,5 \%$ et en $2008,80 \%$ des ménages y possédaient au moins une moto (Benkhelifa, 2009). Il est difficile de chiffrer les mobilités dévolues aux loisirs à Hồ Chí Minh Ville, car peu d'enquêtes y ont été menées. D'après celle dirigée par Patrick Gubry (Gubry et alii, 2008), les distractions représentent 5,1\% des motifs de déplacement, tout niveau social confondu, auxquelles s'ajoute une partie des visites à la famille et aux 
amis $(8,4 \%)$, préférentiellement le week-end et le mercredi. Ces mobilités restent discriminantes socialement : celles des riches ménages représentent le quadruple de celles des pauvres et le double de celles à revenus intermédiaires (Gubry et Linh, 2010). Elles restent également genrées : si 10,2\% des hommes déclarent aller au bar tous les jours après le travail (en particulier au bia hơi, bar populaire où l'on boit de la bière), c'est seulement le cas de $0,7 \%$ de femmes.

Les lieux et les activités de loisirs ne font pas l'objet d'une définition très précise en vietnamien : ils sont couramment désignés par l'expression đi chơi (s'amuser), le terme de giải trí (giải signifiant libérer et trí, l'esprit) ou encore celui de thời gian rỗi (littéralement le temps libre). En outre, les pratiques ne sont pas toujours associées à des lieux spécifiquement dédiés. Ainsi, on peut jouer au badminton dans des interstices urbains, tels des cours de pagode, à la frontière entre public et privé (Miao, 2001). Cette qualification imprécise des lieux et des pratiques témoigne précisément de leur émergence et de leur autonomisation progressive dans des espaces urbains extrêmement denses ${ }^{5}$.

Cherchant à comparer les périmètres spatiaux parcourus par nos enquêtés à partir de leurs lieux de résidence et de travail à des fins de loisirs, nous avons cartographié la localisation de leurs pratiques de loisirs. Như et Nam, d'âges et de revenus différents, donnent ainsi à voir des spatialités variées (illustration 3). Etudiante en architecture de 24 ans, Như pratique essentiellement des loisirs à proximité de chez elle (cafés, karaokés, boutiques et piscine). Ayant à disposition une vieille moto, elle se rend plus exceptionnellement en centre-ville, notamment pour suivre un cours de cuisine et fréquenter des cafés plus à la mode, comme les Highlands Coffee, chaîne vietnamienne. Pour Nam en revanche, trentenaire vice-directeur d'une entreprise de construction, le centre-ville est le lieu privilégié de ses loisirs après le travail (cafés, restaurants). Propriétaire d'une voiture, il emmène régulièrement sa famille le week-end dans le nouveau quartier de Saigon South. Il n'envisage pas son quartier de résidence comme un lieu propice à la détente. 


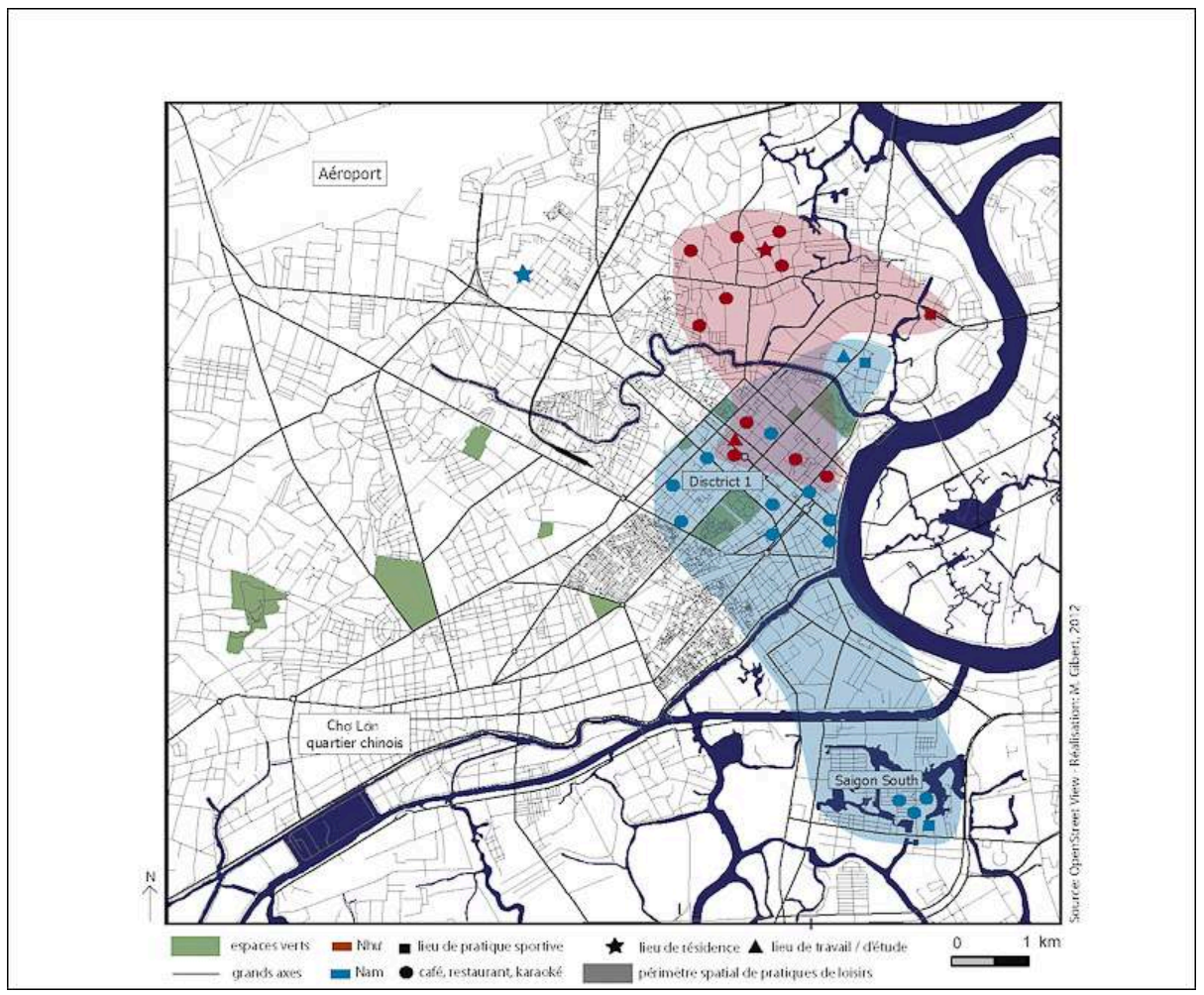

18 Se déplacer est également pensé comme un loisir en tant de tel. La promenade et la marche sont appréciées : 6,1 \% la pratiquent quotidiennement loin devant le théâtre, le cinéma et les concerts (Gubry et alii, 2008). On assiste également à une réinvention de la figure du "flâneur ", telle que pensée par Walter Benjamin, mais à moto. Circuler sans but précis en ville permet de l'explorer et de faire l'expérience de sa «modernité ». Ces flâneries offrent un cadre de découverte renouvelé de la ville, participant de son appropriation et de la construction des identités citadines. Entre mouvement et station, la moto peut également être utilisée comme banc, sur lequel on peut se reposer ou contempler confortablement un paysage. Le soir venu, elle sert aussi une forme d'intimité appréciable pour les jeunes couples et amis.

\section{L'ambivalence du rapport à la ville lue par les loisirs}

Les mobilités de loisirs construisent un rapport ambivalent à la ville, témoignant des compétences de ses habitants à profiter de ses opportunités tout en mettant à distance ses contraintes.

Si les lieux de loisirs se sont multipliés dans les vingt dernières années, ils se concentrent essentiellement en centre-ville. Laboratoire de modernité par excellence, d'abord coloniale puis contemporaine, il est aujourd'hui fréquenté pour son prestige et ses aménités (espaces verts, malls, cafés...). La pression foncière qui s'y exerce le rend inhabitable pour de nombreux citadins. Par les loisirs, ils renégocient cependant leur accès à la centralité urbaine. Nos enquêtés le mentionnent tous comme lieu privilégié de rendez-vous, dont l'expérience est subtilement valorisée. Alors que Hoa, étudiante de 23 ans, circule souvent en bộ đồ ở nhà (sorte de pyjama) dans son quartier, elle n'envisage pas de se rendre en centre-ville sans un effort vestimentaire, ce qui 
participe également de son plaisir. Siroter un café glacé constitue un moyen de négocier pour un bugdet raisonnable une présence prolongée dans le district 1 , qu'elle immortalise souvent avec ses amies par des photos, publiées ensuite sur des réseaux sociaux. Il s'agit pour elle de se mettre en scène, en tant que citadine, dans ces espaces de centralité. Cette expérience est d'autant plus valorisante qu'elle marque son intégration dans une ville qu'elle habite depuis cinq ans seulement pour ses études.

21 Mais fuir le bruit, la pollution, la promiscuité et le manque d'espaces verts des quartiers centraux constitue également un motif de mobilité récréative. Nam apprécie ses escapades à Saigon South car ce quartier incarne selon lui « la ville idéale». Lancée à la fin des années 1990 à une dizaine de kilomètres du centre-ville, cette opération urbaine se singularise par son architecture verticale, ses espaces verts et sa réglementation plus stricte. Nam apprécie de pouvoir s'y promener au calme en famille - la poussette de son fils n'étant pas ici problématique, contrairement aux trottoirs du centre-ville. L'achat d'une voiture a joué un rôle fondamental dans l'extension de son périmètre récréatif, complexifiant son rapport à la ville, tantôt fréquentée pour son effervescence, tantôt mise à distance pour plus de calme.

\section{Mobilités récréatives, mobilités sociales}

A Saigon South, Nam met temporairement à distance son quartier d'habitation plus populaire, dans lequel il s'est installé bien avant son ascension sociale. Évoluant aujourd'hui dans plusieurs cercles de sociabilité de niveaux sociaux différents, il articule par les loisirs les multiples facettes de son identité citadine. Du bia hơi avec ses voisins aux parties de tennis entre collègues, Nam se construit tel un « homme pluriel » (Lahire, 1998). Les loisirs constituent ainsi une clé de lecture stimulante pour appréhender les recompositions socio-spatiales à l'œuvre dans le processus d'émergence. Elles relèvent de fines négociations personnelles oscillant entre ouverture et fermeture, sociabilités de rue et entre-soi sélectif, public et privé.

Plus directement, les loisirs peuvent être mis au service d'une ascension sociale, lorsqu'ils représentent des sésames de milieux socialement et spatialement fermés, tels les clubs de golf ou de tennis. Les dispositifs socio-spatiaux de filtrage y sont en effet nombreux : accès régulé par l'achat d'une carte de membre nécessitant un parrainage, localisation périphérique de clubs, fonctionnant tels des enclaves privées et sécurisées (barrière, végétation occultante, gardiens, vidéosurveillance...). Ces multiples discontinuités spatiales servent plus globalement un entre-soi social valorisant, comme le montre l'exemple de Ngan. Depuis qu'elle travaille comme assistante dans l'audiovisuel, où elle se fait appeler Alison, cette dernière fréquente le Him Lam Golf Club, à quelques kilomètres du centre-ville (illustration en annexe), à raison de deux fois par semaine. Sa carte de membre, initialement délivrée pour jouer avec des clients, sert plus globalement une stratégie de distinction, comme en témoignent ses mises en scène photographiques sur Facebook (illustration 4). Le statut de ces photos est en luimême révélateur de cette négociation subtile entre privé et public dans le cadre de pratiques de loisirs. Publiées sur son profil accessible à tous, du moins à tous ceux qui ont détourné l'interdiction de ce réseau social par le gouvernement, ces photos la montrent pleinement intégrée dans un espace normalement à l'abri des regards. 
Illustration 4 - Pratiques de loisirs et mise en scène sociale : I'exemple d'Alison au Him Lam Golf Club

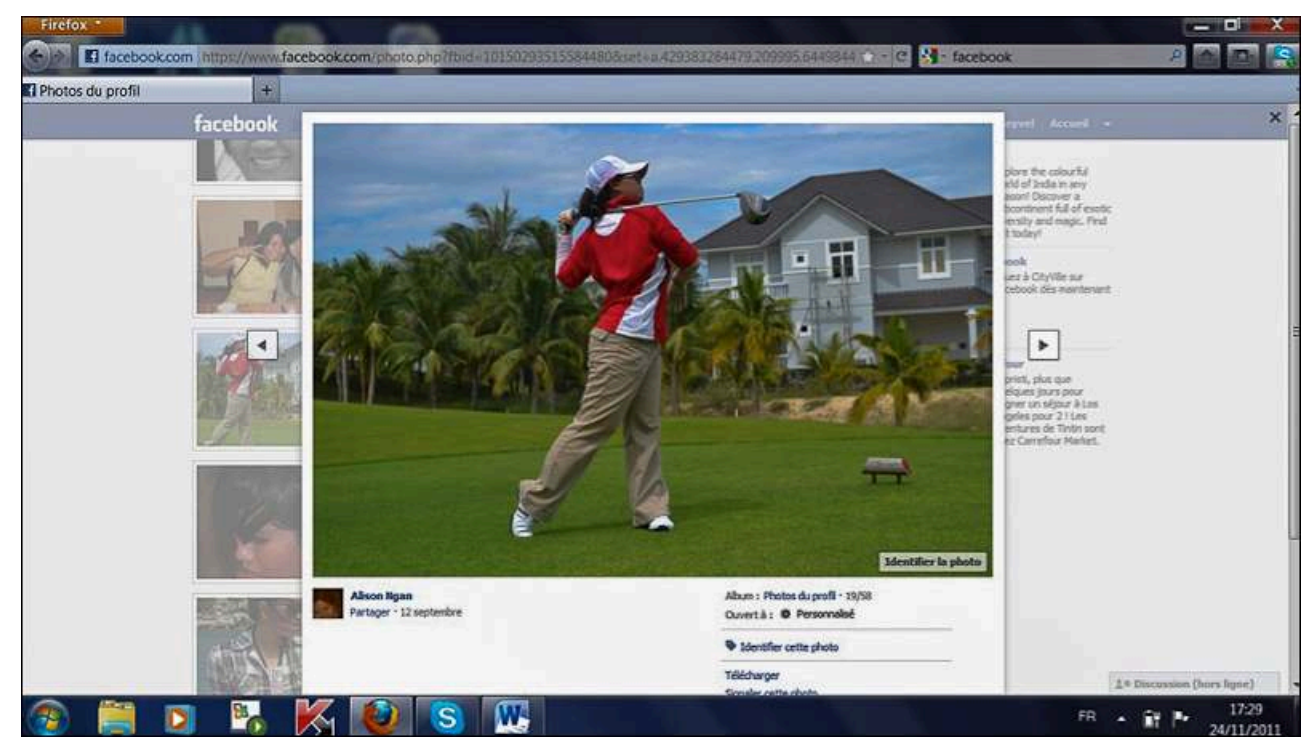

Source: Facebook, 2011.

Les loisirs participent ainsi de la construction d'identités citadines socialement et spatialement différenciées.

\section{La privatisation et le contrôle des loisirs en question}

\section{Une production de plus en plus privatisée des espaces de loisirs en ville}

La montée en puissance du secteur privé dans les activités de loisirs va de pair avec la privatisation croissante des espaces dédiés aux loisirs, en particulier dans les récentes opérations d'urbanisme, pensées en rupture avec la ville héritée. Si la gestion foncière et la planification urbaine relèvent des autorités publiques, la conception et la production des nouveaux quartiers sont de plus en plus confiés à des acteurs privés, souvent étrangers (singapouriens, taïwanais et coréens notamment). Conçus selon des standards internationaux, induisant une organisation fonctionnaliste de l'espace et une réglementation plus stricte des usages, les lieux dédiés aux loisirs et à l'entertainment y trouvent une place centrale mais plus normée. La pratique du sport se limite ainsi à des complexes spécifiquement dédiés, privatisés et payants (illustration 5). Même les parcs, d'apparence ouverte, sont en réalité des espaces privés ouverts au public, soumis à des règlements et des contraintes horaires. Tel est le cas dans le nouveau quartier à la mode du Crescent, pensé comme la centralité récréative de Saigon South. Un ensemble de boutiques et cafés franchisés y sont ainsi regroupés autour d'un plan d'eau, le long duquel a été aménagée une promenade. 


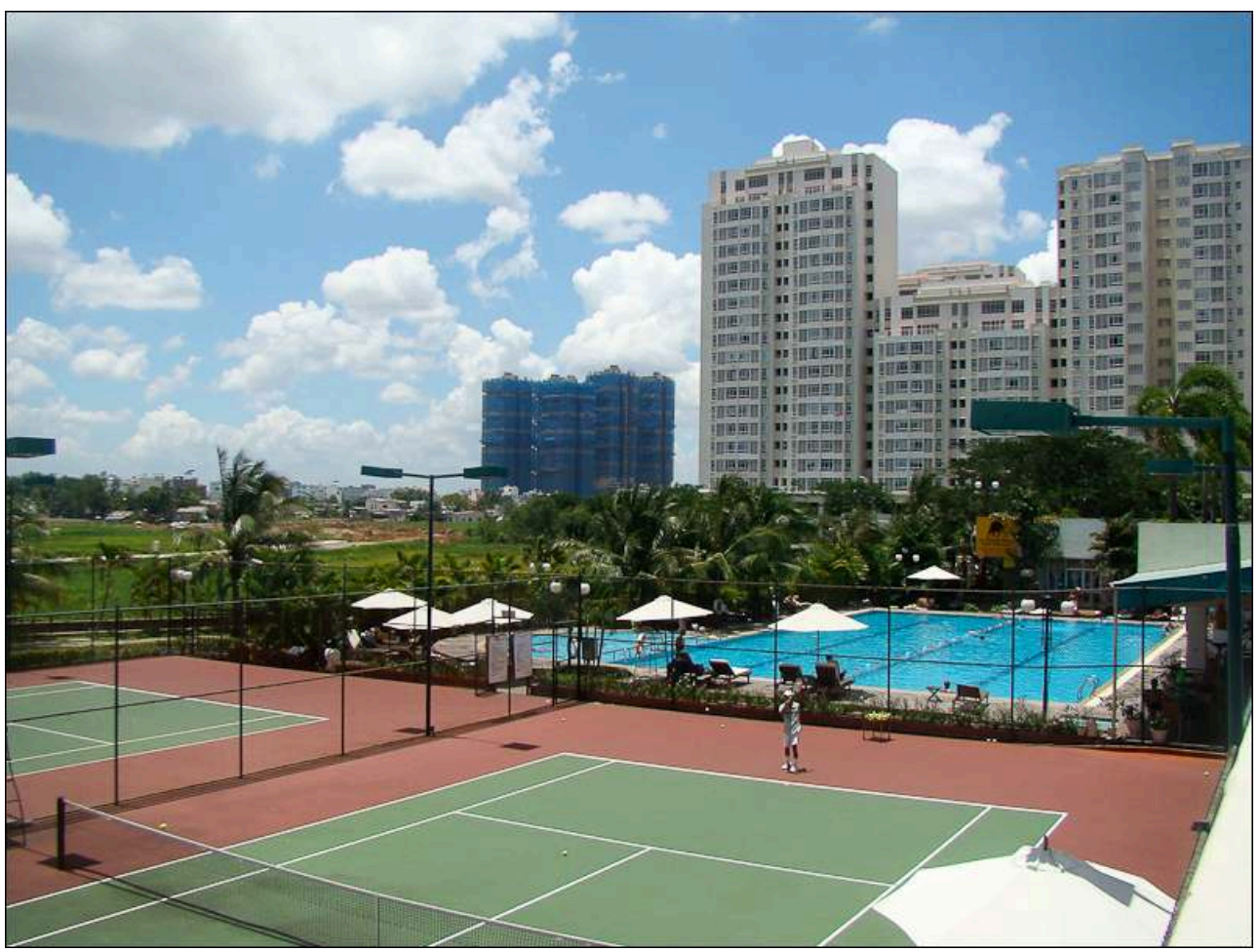

Auteur : M. Gibert, 2011

Ces nouvelles productions urbaines illustrent la convergence, a priori paradoxale, entre les intérêts de la classe moyenne nouvellement propriétaire, souhaitant voir borner et protéger ses biens de tout usage public, et la volonté des autorités urbaines d'organiser un contrôle plus strict de l'espace urbain, en vue de sa modernisation et de sa valorisation (Harms, 2009). En témoigne la proximité frappante entre les affiches de propagande, vantant l'émergence d'une ville dite "civilisée et moderne » (đô thị văn minh, hiện dậi), et le marketing urbain développé dans les publicités des promoteurs, à l'image du slogan de Saigon South: " a communaly cultural lifestyle in a civilized city». La société de consommation des loisirs participe bien à une société du consensus politique (Drummond et Thomas, 2003).

\section{Privatisation et mise en scène publique des pratiques de loisirs}

Saisir les pratiques de loisirs par leurs spatialités permet également de s'interroger sur leur privatisation croissante à l'échelle domestique. Celle-ci peut être interprétée de différentes manières, au rang desquelles figure la hausse du niveau de vie. A mesure que celui d'un ménage augmente, il a davantage la capacité de privatiser une pratique, auparavant exercée dehors, de manière partagée. C'est le cas d'internet et des jeux en réseaux, pratiqués dans les cybercafés, jusqu'à l'achat d'un ordinateur familial. Mais la pratique privée de loisirs peut aussi en permettre l'accès aux plus modestes. L'achat de dvd pirates ou le téléchargement de films à la maison autorise leur visionnage, autrement inaccessible dans de coûteux cinémas. D'autres pratiques de loisirs connaissent des trajectoires spatiales plus complexes encore, à l'image de leur mise en scène négociée entre espaces publics et privés. Le cas du karaoké est à ce titre intéressant. Apparu d'abord dans des cafés, où il devait être partagé entre les clients, il 
peut désormais faire partie de l'équipement domestique. Il profite alors à un cercle de sociabilité plus choisi : la famille, les amis ou encore des voisins de la ruelle. Il peut cependant continuer de représenter une sortie dans des établissements marchands, comme la chaîne Nice Karaoké, proposant des salons particuliers. Les chansons s'y renouvellent plus fréquemment, sur un équipement hi-fi de meilleure qualité, permettant les concours. Chanter s'accompagne d'autres pratiques de plaisir : boire, manger, plaisanter et même séduire, ces salons procurant une intimité appréciable pour de jeunes couples.

La complexité de ces trajectoires spatiales montre également que la privatisation n'est pas systématique à mesure que les moyens des ménages augmentent. Au contraire, certaines pratiques prennent fondamentalement sens à l'extérieur, en ce qu'elles donnent à voir de la richesse. C'est le cas du goûter d'anniversaire organisé pour la jeune Mỹ dans un des KFC de la ville (illustration 6). Offert par ses parents pour ses 10 ans, il a tout autant permis à sa dizaine d'amis de s'amuser sur l'aire de jeu du fast food, en dégustant des ailerons de poulet arrosés de coca cola, que de valoriser la réussite de sa famille aux yeux des autres parents. Nous pourrions développer un raisonnement similaire à propos de la gymnastique : entre le tập thể dục pratiquée tôt le matin dans les parcs et celle des salles de sport, la pratique est la même, mais le changement de lieu indique une évolution sociale du sens qui lui est affecté.

Illustration 6 - Mỹ, 10 ans, fête son anniversaire au KFC

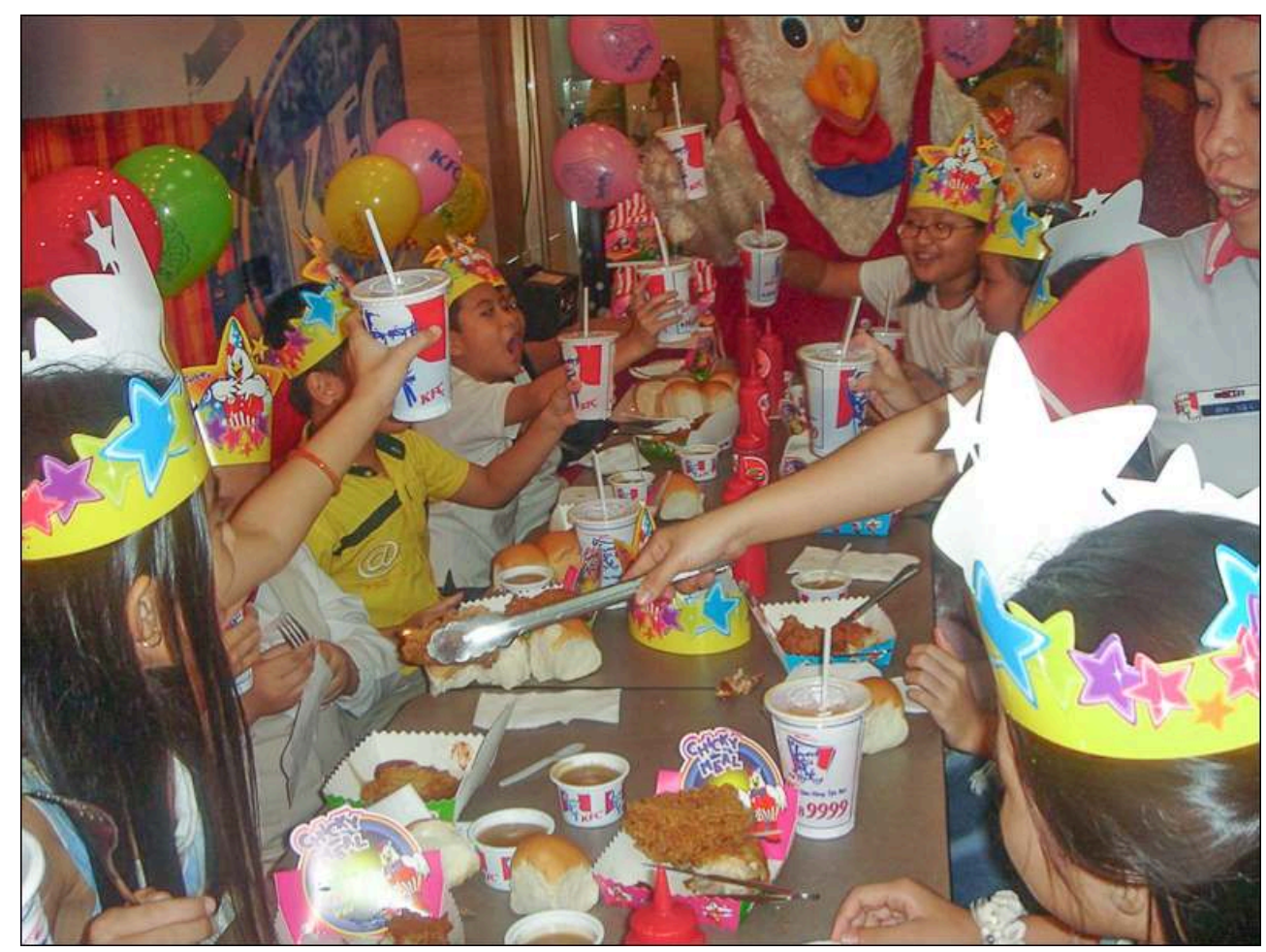

Auteur : E. Peyvel, 2006

Dans un contexte d'individualisation croissante des pratiques, les loisirs apparaissent ainsi à l'interface entre expériences individuelles et collectives, privées et publiques. Moments de relâchement tout autant que de mise en scène, les loisirs constituent également un vecteur dans l'apprentissage de nouvelles normes urbaines. 


\section{Devenir urbain à travers des loisirs policés} processus de civilisation (Elias, 1974). Si la sphère récréative peut être lue selon un relâchement des normes sociales, elle n'en sert pas moins un apprentissage efficace de codes urbains, rappelés par des règlements clairement affichés, concernant désormais autant les espaces privés que publics. Ainsi, à Yoga living, les retards sont sanctionnés par une interdiction d'accès aux cours, car « rubber time is not a practice at Yoga Living ». Par ailleurs, les interdictions de manger, parler, fumer, téléphoner participent d'une injonction à la courtoisie : «It is yoga, please come with a positive mind for yourself and for all ». Cette discipline amène une rigidification de la pratique même du temps libre. Elle est pourtant vécue par les usagers comme un gage de sérieux et de qualité. L'encadrement imposé aux temps libres participe plus globalement à la mise en ordre spatiale et sociale des usages de la ville, au service d'un apprentissage de supposés codes urbains. Les affiches exhortant la population à ne pas cracher, uriner, déambuler en pyjama ou dégrader les équipements publics participent d'une vaste campagne de propagande orchestrée par la ville et intitulée « Réaliser les quartiers culturels » (Làm khu phố văn hóa). Si les règles sont respectées dans le quartier, celui-ci se voit décerner un panneau d'honneur à son entrée.

Illustration 7 - Propagande et mise en valeur des « quartiers culturels »

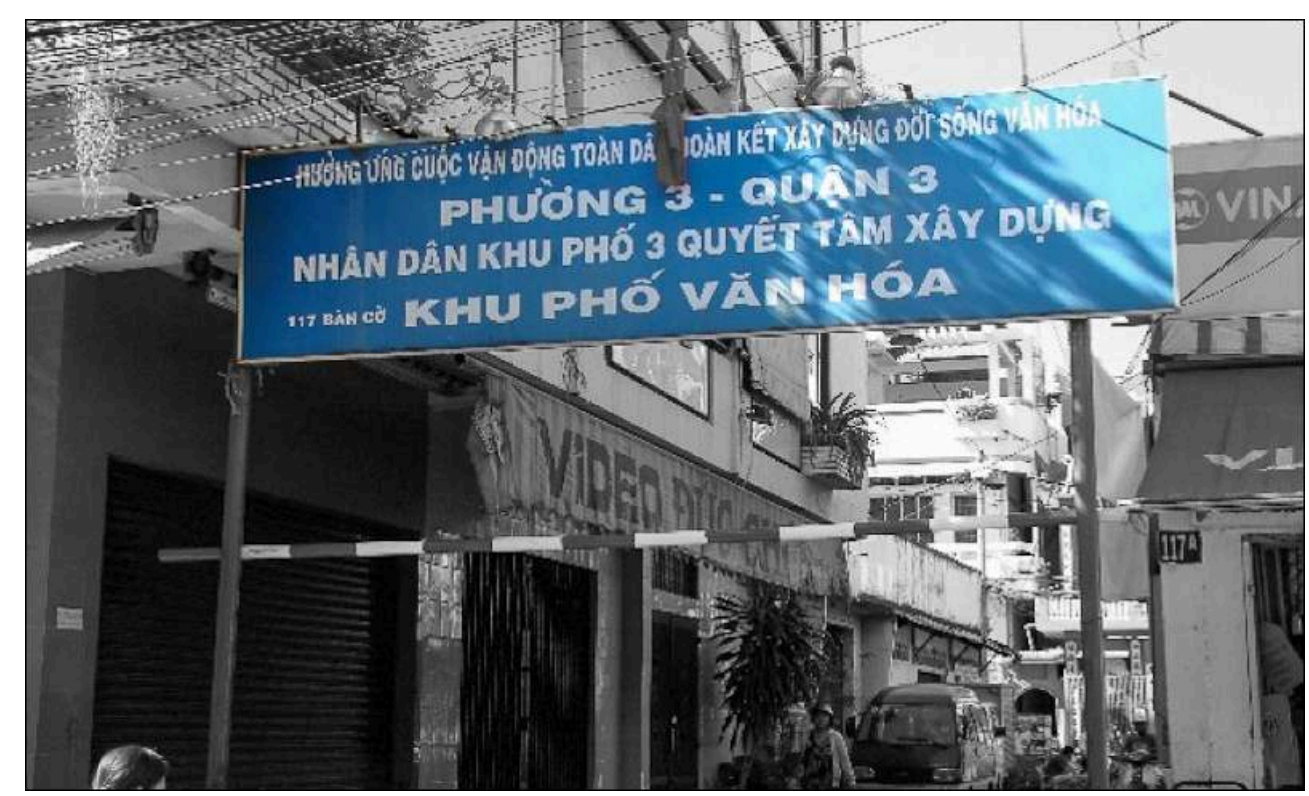

Auteur: M. Gibert, 2009.

31 Ces codes urbains, imposés par un régime autoritaire, participent de la construction d'une ville rêvée, à la croisée de la formation de l'Homme Nouveau socialiste et de l'internationalisation de modèles urbains. 


\section{Conclusion}

32 Analyser les lieux et pratiques de loisirs à Hồ Chí Minh Ville offre un point de vue privilégié pour lire ce qui est communément appelée l'« émergence » du Việt Nam, et mieux comprendre quel sens les populations affectent aux richesses nouvellement produites.

L'étude des conditions d'émergence d'une sphère du bon temps permet tout d'abord de relativiser son caractère nouveau, l'ouverture à des modèles mondialisés de récréation y étant ancienne. Plus que la simple résultante d'un miracle économique, les pratiques de loisirs donnent à voir l'urbanisation, la tertiarisation et la salarisation dans leurs conséquences sociales et mentales, notamment dans la gestion d'un temps libre, structurable et consommable en tant que tel.

D'autre part, si la privatisation croissante des lieux de loisirs à Hồ Chí Minh Ville est indiscutable, ces derniers s'inscrivant de plus en plus dans la sphère marchande, le désengagement de l'État reste relatif. Il est intéressant de remarquer le relatif consensus qui s'établit aujourd'hui entre autorités publiques et acteurs privés concernant la production et la réglementation des espaces de loisirs, au service d'une citadinité idéale. De ce fait, les loisirs offrent un point de vue renouvelé dans la compréhension de cette économie de transition vietnamienne.

En outre, l'étude socio-spatiale des loisirs constitue un vecteur original pour comprendre la construction des identités citadines, la façon dont les habitants négocient leur accès à la ville et donnent à voir leur intégration urbaine. Au-delà, les loisirs sont une clé de compréhension opérationnelle pour mieux saisir la construction des individualités vietnamiennes, dans le plaisir qui leur est accordé.

\section{BIBLIOGRAPHIE}

Baudrillard J., 1970. La société de consommation : ses mythes ses structures. Paris, SGPP, 298 p.

Benjamin W., 1989. Paris, capitale du XIX ${ }^{e}$ siècle : le livre des passages. Paris, Éditions du Cerf, 974 p.

Benkhelifa F., 2008. Une tentative de vision intégrée énergie-urbanisme-environnement : l'expérience de Ho Chi Minh Ville. Actes de la conférence internationale sur les politiques d'efficacité énergétique au Vietnam, Ho Chi Minh Ville, AFD, p. 86-91.

Bourillon F., 2000. Les loisirs et la ville : espaces, institutions, pratiques. Paris, MSH, 222 p.

Castiglioni F., Cusset J.M. et Gubry P. (dir.), 2006. La ville vietnamienne en transition. Hanoi, Institut des Métiers de la Ville, $313 \mathrm{p}$.

CB Richard Ellis Vietnam, 2011. MarketView, Ho Chi Minh City. Ho chi Minh City, CB Richard Ellis Research and Consulting, $8 \mathrm{p}$.

CCIVF (Chambre de Commerce et d'Industrie Française au Vietnam,), 2009. Dossier spécial Vietnam 2009. CCIFV, 15 p. 
Central Population and housing census steering committee, 2010. The 2009 Vietnam Population and Housing Census, Completed Results. Hanoi, Statistical publishing house, 903 p.

Cling J.P., Marouani M.A., Razadindrakoto M., Robilliard A.S. et Roubaud F., 2009. The Distributive Impact of Vietnam's Accession to the WTO. International Economics, vol. 2, nº 118, p. 43-71.

Coll., 2010. Encyclopédie de l'état du monde : histoire, économie, géopolitique. Paris, La découverte.

Delattre S., 2000. Les douze heures noires : la nuit à Paris au XIX'e siècle. Paris, Albin Michel, 674 p.

Drummond L., 2000. Street Scenes: Practices of Public and Private Space in Urban Vietnam. Urban Studies, vol. 37, $\mathrm{n}^{\circ} 12$, p. 2377-2391.

Drummond L., Thomas M. (dir.), 2003. Consuming Urban Culture in Contemporary Vietnam. London, Routledge Curzon, $248 \mathrm{p}$.

Dumazedier J., 1962. Vers une civilisation du loisir ? Paris, Éditions du Seuil, 264 p.

Dumazedier J., Ripert A., 1966. Le loisir et la ville. Tome 1: Loisir et culture. Tome 2: Société éducative et pouvoir culturel. Paris, Seuil, 397 p. et 298 p.

Durand M., 2011. Imagerie populaire vietnamienne. Paris, EFEO, 455 p.

Earl C., 2010. Vietnam's « Informal Public » Spaces: Belonging and Social Distance in Post-reform Ho Chi Minh City. Journal of Vietnamese Studies, vol. 5, $\mathrm{n}^{\circ}$ 1, p. 86-124.

Earl C., 2004. Leisure and Social Mobility in Ho Chi Minh City, in Taylor P., Social Inequality in Vietnam and the Challenges to Reform. Singapore, ISEAS Publications, p. 351-379.

Elias N., 1973. La civilisation des mœurs. Paris, Calmann-Lévy, 447 p.

Gainsborough M., 2000. Political change in Vietnam: In search of the Middle-Class Challenge to the State. Asian Survey, vol. 42, n ${ }^{\circ}$ 5, p. 694-707.

Gubry P., Linh H.P., 2010. Niveau de vie et déplacements dans les métropoles vietnamiennes : Hô Chi Minh ville et Hanoi. Tiers Monde, vol. 1, n 201, p. 107-129.

Gubry P., Lê T.H., Nguyen T.T., Pham T.H., Tran T.T.T. et Vu H.N. (dir.), 2008. Bouger pour vivre mieux : les mobilités intra-urbaines à Hô Chi Minh Ville et Hanoi. Hanoi, Editions de l'Université nationale d'économie, $291 \mathrm{p}$.

Harms E., 2009. Vietnam's Civilizing Process and the Retreat from the Street: A Turtle's Eye View From Ho Chi Minh City. City \& Society, vol. 21, n² 2, p. 182-206.

Ho Chi Minh City Statistical Office, 2010. Statistical Year Book of Ho Chi Minh City. Ho Chi Minh City, Statistical publishing house, $343 \mathrm{p}$.

Khang T., 2007. Ho Chi Minh City [Saigon]: Before the Doorstep of Global Assimilation, in Lim S.W., Asian Alterity With Special Reference to Architecture and Urbanism through the Lens of Cultural Studies. Singapore, World Scientific Publishing Company, p. 20-27.

Khanh C., Tran H., 1978. Le « cercle européen » depuis la libération. Vietnam, nº 232, n.p.

King V.T., 2008. The Middle Class in Southeast Asia: Diversities, Identities, Comparisons and the Vietnamese Case. The International Journal of Asia-Pacific Studies (IJAPS). Vol. 4, nº 2, p. 75-112.

Knafou R., 1997. Tourisme et loisirs. Paris, la Documentation française, coll. « Atlas de France », $\mathrm{n}^{\circ} 7,126 \mathrm{p}$.

Lahire B., 1998. L'homme pluriel : les ressorts de l'action. Paris, Nathan, 271 p. 
Lanfant M.F., 1972. Les théories du loisir : sociologie du loisir et idéologie. Paris, PUF, 256 p.

Lemarchand N., 2008. Géographie du commerce et de la consommation : les territoires du commerce distractif. Habilitation à diriger des recherches en 2 volumes, Paris, Université Paris IV, 220 p. et $142 \mathrm{p}$.

Lloyd K., 2003. Contesting Control in Transitional Vietnam: The Development and Regulation of Traveller Cafés in Hanoi and Ho Chi Minh City. Tourism Geographies, vol. 5, nº 3, p. 350-366.

Marx K., 1894. Le Capital, critique de l'économie politique, Tome 3 : le procès de la production du Capital. Paris, Messidor, n.p.

Miao P. (dir.), 2001. Public places in Asia Pacific cities : Current Issues and Stratégies. Dordrecht, Kluwer Academic Publishers, $391 \mathrm{p}$.

Nguyen V.K., 1995. La société vietnamienne face à la modernité, le Tonkin de la fin du XIXème siècle à la seconde guerre mondiale. Paris, L'Harmattan, $432 \mathrm{p}$.

Peyvel E., 2009. L'émergence du tourisme au Việt Nam, lieux, pratiques et imaginaires. Thèse de doctorat en géographie, Université de Nice Sophia Antipolis, 405 p.

Pinol J.L., 1992. Le monde des villes au XIXe siècle. Paris, Hachette, 230 p.

Sanjuan T. (dir.), 2003. Les grands hôtels en Asie modernité, dynamiques urbaines et sociabilité. Paris, Publications de la Sorbonne, $272 \mathrm{p}$.

Schweyer A.V., 2005. Le Viêtnam ancien. Paris, Les Belles Lettres, 319 p.

Storch H., Eckert R. et Pfaffenbichler P., 2008. The compactness of urban areas in Vietnam. Sustainable urban development and local mobility nodes. Real Corp 008: Mobility nodes as innovation Hubs, Vienna, 19-21 May 2008, 10 p.

Thomas M., 2001. Public Spaces/Public Disgraces: Crowds and the State in Contemporary Vietnam. Journal of Social Issues in Southeast Asia (SOJOURN), vol. 16, $\mathrm{n}^{\circ}$ 2, p. 306-330.

Truitt A., 2008. On the Back of a Motorbike: Middle-class Mobility in Ho Chi Minh City, Vietnam. American Ethnologist, vol. 35, $\mathrm{n}^{\circ}$ 1, p. 3-19.

Waibel M., 2006. The Production of Urban Space in Vietnam's Metropolis in the Course of Transition : Internationalization, Polarization and Newly Emerging Lifestyles in Vietnamese Society. Trialog, vol. 2, no 89, p. 43-48.

http://www.the-crescent.com

http://www.saigonsouth.com

http://www.saigon-tourist.com

http://www.yogaliving.com.vn/site

\section{NOTES}

1. En 2008 , le taux d'alphabétisation était de $90,3 \%$ et l'espérance de vie moyenne atteignait les 71 ans (CCIFV, 2009).

2. Voir notamment : Castiglioni, Cusset et Gubry (dir.), 2006 ; Khang, 2007 ; Waibel, 2006.

3. Le recensement officiel ne prend pas en compte la population dite «flottante», non enregistrée. On estime en réalité la population de Hồ Chí Minh Ville à près de dix millions d'habitants. 
4. Le terme anglophone de «mall » désigne un complexe commercial fermé associant à la fois des boutiques mais également des espaces de restauration, de déambulation et de loisirs (cinéma, bowling...). Il s'agit d'un espace intégré, plus complet que le centre commercial. A ce sujet, voir notamment les travaux de Nathalie Lemarchand (Lemarchand, 2008).

5. La densité moyenne dans les districts urbains de Hồ Chí Minh Ville s'élevait à $26000 \mathrm{hab} / \mathrm{km}^{2}$ en 2006 (Storch, Eckert et Pfaffenbichler, 2010).

\section{RÉSUMÉS}

Cet article propose d'envisager l'émergence du Việt Nam par une approche socio-spatiale des pratiques de loisirs à Hồ Chí Minh Ville. Celle-ci constitue une façon concrète d'approcher les usages de la richesse nouvellement produite, sa répartition et le sens qui lui est affecté. En outre, les loisirs offrent un point de vue original sur la fabrique urbaine, la construction des identités citadines et la façon dont les habitants négocient leur accès à la ville, dans un contexte renouvelé d'enrichissement et d'urbanisation. Nous posons comme hypothèse que les pratiques de loisirs à Hồ Chí Minh Ville aboutissent à une différenciation accrue des espaces publics et privés depuis la politique du Renouveau, engagée à partir de 1986.

This article aims to approach the emergence of Việt Nam through a socio-spatial analysis of leisure practices in Hồ Chí Minh City. Studying leisure practices is tangible way to analyze the way recent national wealth is put to use, its distribution and social meaning. Furthermore, leisure activities offer an original viewpoint on the construction of urban identities and on how citizens negotiate their access to the city in the new context of urbanization and economic enrichment. Our hypothesis is that leisure practices in Hồ Chí Minh City generate a growing differentiation between public and private spaces since the renovation engaged in 1986.

\section{INDEX}

Keywords : access to the city, emergence, Hồ Chí Minh City, leisure, privatization, public spaces, urban identities, Việt Nam

Mots-clés : accès la ville, émergence, espaces publics, Hồ Chí Minh Ville, identité citadine, loisirs, privatisation, Việt Nam

Thèmes : Sur le Champ - Sur le Terrain

\section{AUTEURS}

\section{EMMANUELLE PEYVEL}

Emmanuelle Peyvel, empeyvel@yahoo.fr, est maître de conférences en géographie à l'Université de Bretagne Occidentale et membre de l'Institut d'Asie Orientale (IAO), UMR 5062, Lyon. Elle a publié récemment :

- Peyvel E., 2011. Visiting Indochina, the imaginary of the French colonial period in today's touristic Việt Nam. Journal of tourism and cultural change, vol. 9, n³ 3, p. 226-236. 
- Cabasset C., Peyvel E., Sacareau I. et Taunay B., 2009. De la visibilité à la lisibilité : le tourisme domestique en Asie. Quelques réflexions à partir des cas chinois, indien, indonésien et vietnamien. Espace, Populations, Sociétés, n²-3, p. 221-235.

- Peyvel E., 2009. Mũi Nế (Việt Nam) : deux approches différenciées de la plage par les touristes occidentaux et domestiques. Géographie et Cultures, n 67, p. 79-92.

\section{MARIE GIBERT}

Marie Gibert, marie_gibert@hotmail.com, est doctorante en géographie à l'Université Paris 1 Panthéon-Sorbonne, UMR 8586 PRODIG. Elle a publié récemment :

- Gibert M., 2010. Moderniser la ville, réaménager la rue à Ho Chi Minh Ville. EchoGéo [En ligne], $\mathrm{n}^{\circ} 12$, mis en ligne le 31 mai 2010.

- Gibert M., 2011. Streets as an inclusive public space. A case study : Ho Chi Minh City, Vietnam. The eleventh Asian Urbanization Conference, Osmania University, Hyderabad, Inde, 10-13th December 2011 (Publication des actes à paraître)

- Gibert M., 2011. Local neighbourhood in Ho Chi Minh City, Between spaces of control and spaces of negotiation. Postgraduate and Early Career Symposium " The social dynamics of neighbourhood", Centre for Housing Research, University of St Andrews, Scotland, 23rd May 2011. 